\title{
Reflexiones acerca de la relación entre justicia constitucional y formulación de políticas públicas con enfoque basado en derechos humanos*
}

\section{Reflections about the Relationship between Constitutional Justice and the Proposal of Public Policies with a Human Rights Based Approach}

Alfonso Renato Vargas Murillo** https://orcid.org/0000-0003-4205-2215

http://dx.doi.org/10.21503/lex.v17i24.1810

* Artículo derivado del trabajo de investigación "La participación del juez constitucional en la formulación de políticas públicas con enfoque de derechos humanos: el caso del Tribunal Constitucional Peruano” para optar el título de máster en Gobernanza Global y Derechos Humanos de la Universidad de Castilla-La Mancha, Toledo, España, 2019.

** Máster en Gobernanza Global y Derechos Humanos por la Universidad de Castilla-La Mancha, España. Maestrando en Filosofía con mención en Ética y Filosofía Política por la Universidad Nacional de San Agustín de Arequipa. Título de Especialista en Gobernabilidad, Derechos Humanos y Cultura de Paz por la Universidad de CastillaLa Mancha, España. Bachiller en Derecho por la Universidad Privada de Tacna. Actualmente es consultor e investigador independiente. Tacna, Perú.

Correo electrónico: avargasm95@gmail.com

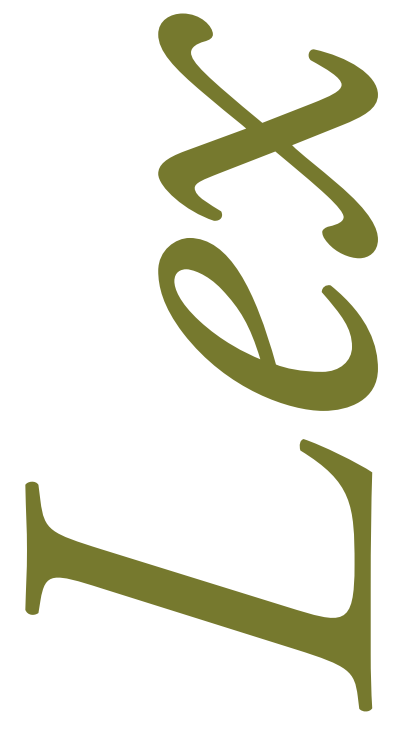




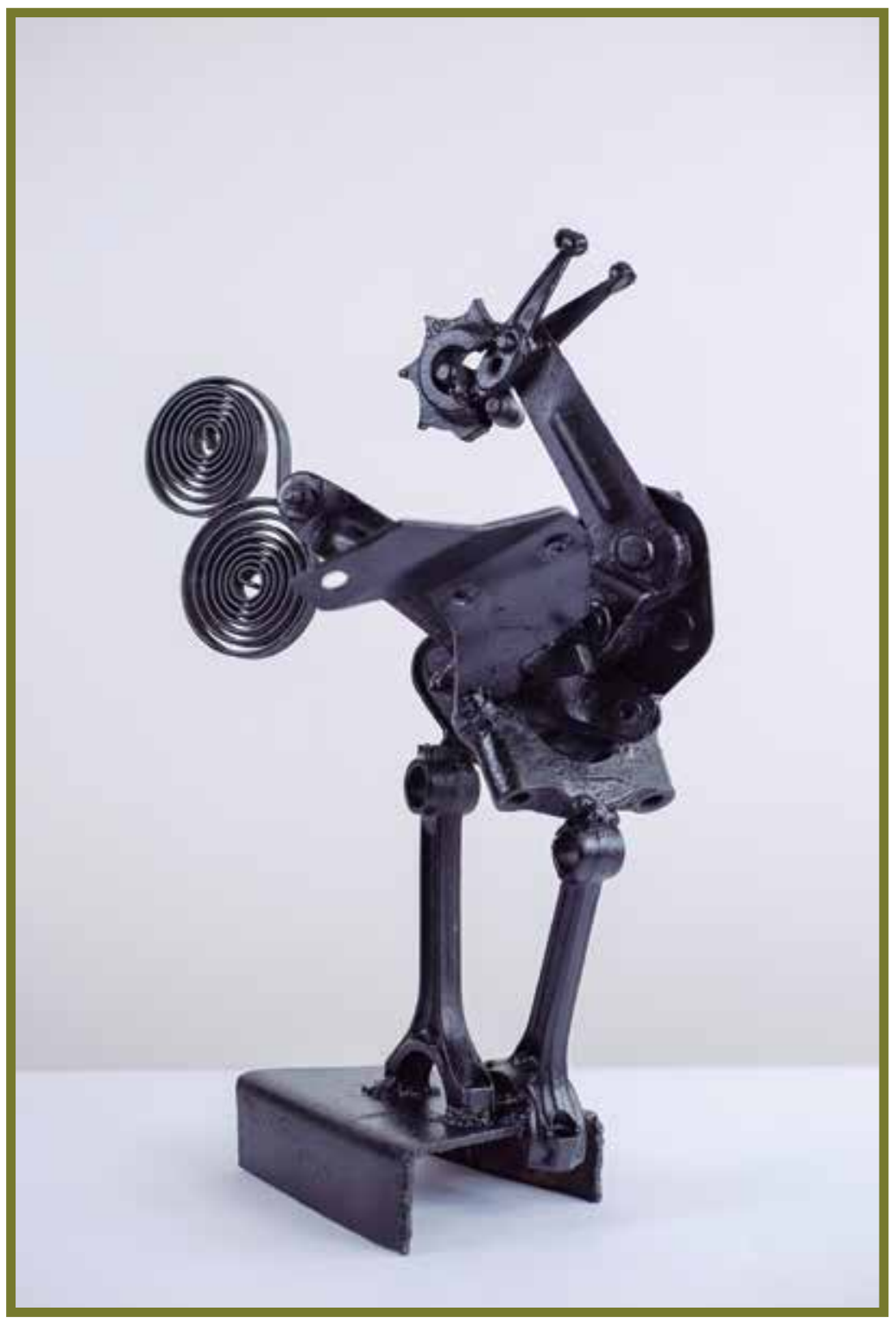

Despertar, 1978. Metal soldado, 55 × $35 \times 35 \mathrm{~cm}$.

Destacado artista plástico nikkei, Oswaldo Higuchi (Perú, 1948) 


\section{RESUMEN}

El presente trabajo pretende describir y explicar brevemente la manera en la que los jueces constitucionales se ven implicados a partir de los planes nacionales de Derechos Humanos en el proceso de formulación de políticas públicas con enfoque de derechos humanos, y los posibles problemas a los que deben enfrentar en este proceso, como los cuestionamientos a su legitimidad democrática.

Palabras clave: jueces, políticas públicas, derechos humanos.

\section{ABSTRACT}

This paper aims to briefly describe and explain how the constitutional judges are involved - as from the Human Rights' national plans - in the process of proposing public policies with a human rights based approach, and the possible problems they should face during this process such as objections to its democratic legitimacy.

Key words: judges, public policies, human rights. 


\section{INTRODUCCIÓN}

Desde finales de siglo XX e inicios del siglo XXI — como apunta Cesar Landa—, se han producido cambios sustanciales en la justicia constitucional en América Latina, lo que se verifica con la instalación de cortes o tribunales constitucionales, las nuevas competencias otorgadas a las Cortes Supremas o la creación de salas especializadas dentro de dichas cortes. Esta situación evidenciaría que el Estado de derecho en América Latina se fundamenta principalmente en la supremacía constitucional y la defensa de los derechos constitucionales, pero que adquiere características particulares en este contexto, toda vez que "dada la experiencia autoritaria, militar o civil, en la región, no puede haber justicia constitucional, sin derecho ni democracia, como tampoco puede haber democracia sin derecho ni justicia constitucional". ${ }^{1}$

Pese a ello, América Latina mantiene condiciones de desigualdad, exclusión a las prestaciones de servicios básicos para la vida digna, conflictos culturales, evidenciando que los Estados del continente no han podido responder a las promesas en materia de derechos contenidas en sus cartas políticas, es decir, no se han traducido en políticas públicas que permitan materializar dichos derechos. ${ }^{2}$

Es en ese marco donde las Cortes (y en especial las altas Cortes o Cortes constitucionales) han tomado un rol más activo en las últimas décadas, desarrollando técnicas como la de Estado de Cosas Inconstitucionales con el fin de frenar la violación masiva de derechos derivadas de la ausencia de condiciones que permitan garantizar dichos derechos por parte de los órganos estatales. Esto se ha visto reforzado por la implementación de los Planes Nacionales de Derechos Humanos, los cuales involucran a todas las entidades públicas en la promoción y participación en el proceso de formulación de políticas públicas con enfoque de derechos humanos. Sin embargo, de la renuencia en muchos casos de las autoridades de implementar lo exhortado en las sentencias de este tipo, con el riesgo de que se conviertan en meras decla-

1 César Landa Arroyo, "Justicia constitucional en América Latina”, Revista Ius et veritas 49 (2014): 109.

2 Christian Steiner, "Presentación”, en Justicia Constitucional y Derechos Fundamentales $N^{\circ}$ 5: La protección de los derechos sociales, las sentencias estructurales, ed. por Víctor Bazán (Bogotá: Konrad Adenauer, 2015), xi. 
raciones de intenciones, surge la necesidad de elaborar mecanismos de control de implementación, pero que permitan mantener su legitimidad dentro del sistema de división de poderes y de frenos y contrapesos.

El presente trabajo pretende describir y explicar brevemente la manera en la que los jueces constitucionales se ven implicados a partir de los planes nacionales de derechos humanos en el proceso de formulación de políticas públicas con enfoque de derechos humanos, y los posibles problemas a los que deben enfrentar, como los cuestionamientos a su legitimidad democrática.

\section{LA JUSTICIA CONSTITUCIONAL EN AMÉRICA LATINA}

Para Aníbal Quiroga, la justicia o jurisdicción constitucional, se puede definir como el "proceso histórico surgido del propio desarrollo constitucional de los Estados modernos de derecho, que establecieron mecanismos de control, autocontrol y de defensa de la supremacía y vigencia constitucional". ${ }^{3}$ De esta manera, no es posible comprender la justicia constitucional en sus diversas dimensiones y expresiones en los países latinoamericanos, sin analizar el lugar que ocupa en el proceso de constitucionalización del ordenamiento jurídico, es decir, el tránsito del Estado de Derecho al Estado Constitucional de Derecho.

La importancia de la justicia constitucional en la construcción del Estado Constitucional de Derecho en los países latinoamericanos se encuentra marcada por la crisis institucional y de representatividad de los parlamentos y las cortes de la jurisdicción ordinaria, lo que la ha convertido en una forma de canalizar y resolver jurídicamente "grandes cuestiones políticas y socio-económicas". ${ }^{4}$ Esto llevaría a establecer y promover una nueva relación entre el Poder Judicial, los otros órganos estatales y la sociedad civil.

Para alcanzar de dichos fines, "los jueces deben controlar y aun suplir a los demás poderes para garantizar los derechos y hacer efectivas las promesas constitucionales", ${ }^{5}$ lo que en un extremo implicaría, incluso, la posibilidad de actuar frente a omisiones legislativas, pudiendo declarar lo que se conoce como inconstitucionalidad por omisión, tomando un posición más activa que la de mero legislador negativo, desarrollando competencias "atípicas" en virtud de los principios de "interdicción de la arbitrariedad y del principio de "fuerza normativa constitucional". 6

Aníbal Quiroga, “La Justicia Constitucional”, Revista Derecho PUCP 41 (1987): 325.

4 César Landa Arroyo, "Justicia constitucional en América Latina”, Revista Ius et Veritas 49 (2014): 110.

5 Alfonso Santiago, "Neoconstitucionalismo", en Anales de la Academia de Ciencias Morales y Politicas - Instituto de Politica Constitucional (Buenos Aires: Instituto de Política Constitucional, 2008), 13.

6 César Landa Arroyo, Organización y funcionamiento del Tribunal Constitucional: entre el derecho y la politica (Lima: Palestra, 2011), 108. 
$\mathrm{Al}$ incrementar la discrecionalidad de las cortes constitucionales, se presenta la gama de nuevas prerrogativas que poseen los tribunales constitucionales ligados a objetivos que resultarían difíciles de conseguir de otro modo en las condiciones de fragilidad institucional de los órganos públicos, inestabilidad política y con políticas públicas incapaces de responder ante las exigencias de los denominados DESC.

Los aspectos característicos de la práctica de las cortes constitucionales en este contexto son los siguientes: carácter operativo de los derechos fundamentales, aplicación del principio "pro-homine" en las controversias normativas relativas a la tutela de derechos, análisis intensivo de razonabilidad respecto a las "libertades preferidas", máxima tutela del derecho a la autonomía y privacidad personal, máxima tutela del derecho a la igualdad en la forma de no discriminación y surgimiento de las "categorías sospechosas", surgimiento de los derechos sexuales, tutela judicial de los derechos colectivos, desarrollo de la declaración de inconstitucionalidad por omisión, obligatoriedad de observar las pautas de los tribunales internacionales, control de convencionalidad, así como la flexibilidad y diversidad de sentencias que dictan los tribunales constitucionales, por ejemplo, aquellas sentencias que establecen recomendaciones y plazos de adecuación a los otros órganos públicos. ${ }^{7}$

Las características mencionadas anteriormente han generado una dinámica particular respecto a la relación entre los poderes públicos en los diversos países latinoamericanos. Es así que la renovación en los enfoques, métodos y técnicas que utilizan los jueces para resolver cuestiones relativas a derechos fundamentales en América Latina, ha obedecido a la identificación de falencias estructurales que impiden la materialización de estos, lo que resulta un impedimento para la construcción de un verdadero Estado Constitucional de Derecho, lo que ha llevado a centrar la atención de la sociedad en las altas cortes como un camino respecto a la formulación de las políticas públicas.

\section{POLÍTICAS PÚBLICAS Y ENFOQUE DE DDHH}

Las políticas públicas pueden definirse como "una estrategia”, en tanto con aquellas el gobierno realiza un conjunto de acciones sucesivas que pretenden articular y coordinar los comportamientos de los actores, y en este sentido "representan la realización concreta de decisiones en torno a uno o varios objetivos colectivos, considerados necesarios o deseables en la medida en que hacen frente a situaciones socialmente relevantes". ${ }^{8}$

7 Alfonso Santiago, "Neoconstitucionalismo", en Anales de la Academia de Ciencias Morales y Políticas - Instituto de Politica Constitucional (Buenos Aires: Instituto de Política Constitucional, 2008), 17-18.

8 Jaime Torres-Melo y Jairo Santander, Introducción a las politicas públicas: conceptos y herramientas desde la relación entre Estado y ciudadanía (Bogotá: IEMP Ediciones, 2013), 56. 
Otra definición, siguiendo a Manuel Quinche-Ramírez y Juan Rivera-Rugeles, plantea que las políticas públicas son un conjunto de acciones diseñadas por el Estado (y ejecutadas directamente por él en ocasiones) con las cuales busca intervenir en situaciones que se han identificado por lo general como socialmente problemáticas, para cumplir ciertos objetivos relativos — usualmente- a la corrección de dichas situaciones. Sin embargo, dichos autores advierten que frente a la existencia de diversas concepciones de las políticas públicas, las cuales pueden resaltar o poner énfasis en algún aspecto respecto a los otros incluso obviando intencionalmente algunas de sus características primordiales, Roth plantea cuatro elementos mínimos para identificar una política pública, los cuales se resumen en: identificar la situación problemática o de interés respecto a la cual se pretende intervenir, definir los objetivos de la intervención, diseńar el plan de acción para lograr los objetivos e identificar la manera en la que participará el Estado (directa o indirectamente). ${ }^{9}$

El Banco Interamericano de Desarrollo ha sostenido que la formulación de políticas públicas comprende "todo el proceso de discusión, aprobación e implementación de las políticas públicas". ${ }^{10}$ La formulación de las políticas públicas se puede dividir en las siguientes etapas: 1) Planteamiento de la agenda: referida a la fase en que la autoridad pública realiza el diagnóstico, recojo de evidencia y se toman decisiones sobre aquello que se va a implementar; 2) Diseńo: referida a los dispositivos, estrategias, programas y recursos para su realización; 3) Implementación: etapa de gestión (producción de bienes y servicios, se realizan control y monitoreo de las actividades); 4) Evaluación: se mide la intervención en base a los criterios de eficacia y eficiencia. ${ }^{11}$

Tradicionalmente se ha sostenido las políticas públicas y los derechos humanos son discursos cualitativamente distintos, toda vez que los primeros se caracterizan por basarse argumentos de tipo operativo o técnico y los segundos de tipo puramente normativo, ${ }^{12}$ por lo que no es posible determinar con claridad su influencia en la formulación de políticas públicas. Sin embargo, es precisamente el carácter normativo, y la vinculatoriedad de las normas constitucionales que contienen derechos, ${ }^{13}$ lo que hace que sea necesaria dicha conexión, en tanto

9 Manuel Quinche-Ramírez y Juan Rivera-Rugeles, "El control judicial de las políticas públicas como instrumento de inclusión de los Derechos Humanos", Vniversitas 121 (2010): 117-118.

10 Banco Interamericano de Desarrollo, La politica de las politicas públicas (Editorial Planeta: México D.F., 2006$), 18$.

11 Renata Bregaglio, Renato Constantino y Carmela Chávez, Políticas públicas con enfoque de derechos humanos en el Perú (Lima: IDEHPUCP - Konrad Adenauer, 2014), 16.

12 Manuel Quinche-Ramírez y Juan Rivera-Rugeles, "El control judicial de las políticas públicas como instrumento de inclusión de los Derechos Humanos", Vniversitas 121 (2010):116.

$13 \mathrm{Al}$ respecto, es necesario señalar, que dicha afirmación se realiza en el marco de lo que Guastini ha denominado "constitucionalización del ordenamiento jurídico", es decir, el camino hacia la construcción de un verdadero Estado Constitucional de Derecho, el cual tiene como uno de sus caracteres "la fuerza vinculante de la Constitución”, respecto de la cual advierte José Rivera: “(...) las constituciones (...) han dejado de ser meras cartas políticas, para constituirse en verdaderas o genuinas normas jurídicas de aplicación directa a las relaciones entre el Estado y los ciudadanos, de aplicación juris- 
por un lado los derechos humanos requieren ser materializados — lo que se puede producir a través de políticas públicas-, y por el otro, las políticas públicas siempre son analizadas, diseńadas y evaluadas a través de un determinado enfoque. Es en ese momento donde el denominado Enfoque Basado en Derechos Humanos (en adelante EBDH) resulta bastante conveniente para poder determinar si las políticas públicas satisfacen las condiciones que impone la normativa relativa a la protección de los derechos humanos. El EBDH, como sostiene la Oficina del Alto Comisionado de Naciones Unidas (OACDH), es un

marco conceptual para el proceso de desarrollo [...] basado en las normas internacionales de derechos humanos, y desde el punto de vista operacional, está orientado a la promoción y la protección de los derechos humanos [...] [y permite] analizar las desigualdades que se encuentran en el centro de los problemas de desarrollo y corregir las prácticas discriminatorias y el injusto reparto del poder que obstaculizan el progreso en materia de desarrollo. ${ }^{14}$

En este sentido, surge la necesidad de elaborar lineamientos —a través de instrumentos legales - que permitan la incorporación de este enfoque en el proceso de formulación de políticas públicas, promoviendo la materialización de los derechos fundamentales reconocidos en el orden nacional e internacional. Es así que, siguiendo las recomendaciones promovidas por las Naciones Unidas desde inicios de la década de los noventa, diversos países empiezan a desarrollar planes nacionales para la incorporación del EBDH en la formulación de políticas públicas. ${ }^{15}$

Por ejemplo, en el caso peruano, se formuló el denominado "Plan Nacional de Derechos Humanos" (PNDH), el cual se define como una herramienta "destinada a asegurar la gestión de políticas públicas en materia de derechos humanos en el país (...) compromete a todos los sectores y niveles de gobierno, y permite proyectar el EBDH en la intervención de las entidades estatales". ${ }^{16}$ La primera versión de este plan se desarrolló para el periodo 2006-2010, siguiendo las recomendaciones del Handbook on National Human Rights Plans of Action (Oficina del Alto Comisionado de la Naciones Unidas, 2002). Dicho plan fue prorrogado hasta el año 2011. Luego de tres ańos, entra en vigencia el segundo plan para el periodo 2014-2016,

diccional. En consecuencia, las normas de la Constitución son genuinas normas jurídicas, vinculantes y susceptibles de producir efectos jurídicos inmediatos". José Rivera, "Fundamentos sobre el carácter vinculante de las resoluciones del Tribunal Constitucional”, Anuario Iberoamericano de Justicia Constitucional 9 (2005): 347.

14 Oficina del Alto Comisionado para los Derechos Humanos, Preguntas frecuentes sobre el enfoque de derecho en la cooperación para el desarrollo (Nueva York y Ginebra: OACHD, 2006), 15. Citado por: Renata Bregaglio, Renato Constantino y Carmela Chávez, Politicas públicas con enfoque de derechos humanos en el Perú (Lima: IDEHPUCP - Konrad Adenauer, 2014), 18.

15 Renata Bregaglio, Renato Constantino y Carmela Chávez, Políticas públicas con enfoque de derechos humanos en el Perú (Lima: IDEHPUCP - Konrad Adenauer, 2014), 31.

16 Ministerio de Justicia y Derechos Humanos del Perú, Plan Nacional de Derechos Humanos 2018-2021, acceso el 10 de enero de 2019,

http://spij.minjus.gob.pe/content/banner_secundario/img/muestra/PLAN-ANUAL.pdf 
el cual — como afirma el propio MINJUSDH— fue formulado a partir de la acentuación de la dimensión participativa contenida en el Handbook on National Human Rights Plans of Action, lo que trajo como resultado cuatro lineamientos estratégicos para cumplir los objetivos identificados en el instrumento anterior. ${ }^{17}$

El PNDH para los años 2018-2021 se aprobó a través del Decreto Supremo 002-2018JUS. Esta versión comprende 5 lineamientos estratégicos, dentro de los cuales identifica trece grupos de especial protección: personas adultas mayores, población afroperuana, personas con discapacidad, mujeres, niños, niñas y adolescentes, personas privadas de libertad, personas con VIH/SIDA y personas con TBC, trabajadoras y trabajadores del hogar, personas LGBTI, defensores y defensoras de derechos humanos, pueblos indígenas, personas en situación de movilidad y personas víctimas de la violencia ocurrida entre los años 1980 y 2000. Este plan al ser transversal, involucra y compromete a todas las entidades públicas, por lo que los jueces se convierten en actores igualmente importantes que otros en la formulación de políticas públicas en base al EBDH.

\section{ACTIVISMO JUDICIAL, POLÍTICAS PÚBLICAS Y EL PROBLEMA DE LA LEGITIMIDAD}

Una primera pregunta que surgió a partir del nuevo rol de las cortes de controlar la constitucionalidad de los actos de los otros poderes, fue la de ¿quién debe ejercer el control constitucional? (identificable en la discusión Schmitt (1931) - Kelsen (1930) sobre quién debe ser el guardián de la Constitución). Luego de identificar el potencial del control constitucional y ante su expansión alrededor del mundo, surge la pregunta de cómo se debe ejercer ese control constitucional. Y por último, ante el reforzamiento de la función judicial y frente a las nuevas prerrogativas otorgadas (y en muchos caso autootorgadas) surge la pregunta: ¿̨hasta dónde se debe extender el poder de los órganos de control jurisdiccional constitucional? ${ }^{18} \mathrm{La}$ respuesta a dicha pregunta dependerá mucho de la teoría de la democracia con la que se lea, así como del lugar que ocupan las cortes en la cultura jurídica de cada país.

Respecto a la relación entre los órganos jurisdiccionales y las políticas públicas, Pavel Corilloclla afirma que existen dos grandes corrientes: la norteamericana y la europea. La primera ha estudiado la labor del Poder Judicial como actor político, es decir, la manera en la que toma estratégicamente sus decisiones, la forma en la que interactúa con otros órganos estatales y grupos de interés que acuden a esta vía para lograr sus objetivos. La europea, se ha enfocado en estudiar los efectos de la actuación de las cortes en la política o el sistema po-

17 Ministerio de Justicia y Derechos Humanos del Perú, Plan Nacional de Derechos Humanos 2018-2021, acceso el 10 de enero de 2019,

http://spij.minjus.gob.pe/content/banner_secundario/img/muestra/PLAN-ANUAL.pdf

18 Omar Sumaria y Karla Vasalla, "Un nuevo actor político: la participación de los tribunales constitucionales en la elaboración de las políticas públicas”, Revista Derecho y Sociedad 40(2013): 124. 
lítico. ${ }^{19}$ Si bien no profundizaremos en ambas corrientes, es necesario tomar en cuenta estos dos enfoques, toda vez que nos permitirá tener una mirada más general de la actuación de las cortes constitucionales en el contexto latinoamericano, analizando su particular forma de ser sin denegar la influencia de los modelos de jurisdicción constitucional en los que se inspira (el modelo norteamericano o anglosajón y el continental o europeo).

Los tribunales constitucionales, como afirma Juan Manuel Sosa (siguiendo a Zagrebelzky y Haberle), son actores políticos en tanto se pronuncian sobre cuestiones consideradas como "res publica", e incluso en tanto cumplen funciones específicas de carácter político y social como "las funciones republicana (de conducción e integración), reconciliadora, moderadora, de reconocimiento e inclusión, y racionalizadora". ${ }^{20}$ Sin embargo, la actuación de las altas cortes nacionales considerada como "activista" ha sido considerada como una extralimitación de poderes, intromisión a la esfera de otros poderes e incluso la desnaturalización de la función jurisdiccional.

Es así que el déficit institucional de la democracia en el país y la continua desatención de demandas sociales han llevado a que estas se trasladen de los foros políticos (en sentido estricto, como el Parlamento) a los foros judiciales. Y si bien estos procesos se inician en muchos casos en la jurisdicción ordinaria, luego son trasladadas a las cortes constitucionales (vía acción de amparo por ejemplo), lo que ha generado un escenario en el que estas cortes se convierten en lo que Haberle denominó "Tribunal de las esperanzas ciudadanas", al cumplir el rol de "activador" de las funciones de los otros órganos públicos. ${ }^{21}$

Si bien el activismo judicial ${ }^{22}$ en muchos casos ha traído como resultado la puesta en agenda y la atención de asuntos públicos muchas veces postergados por la autoridad, las críticas a estas prácticas no han sido pocas y podemos hallarlas, por ejemplo, desde la teoría jurídica en la comprensión formalista del Derecho, la cual considera que

19 Pavel Carilloclla, La justicia constitucionaly las politicas públicas: una aproximación (Santiago de Chile, Universidad Diego Portales, 2008), 5.

20 Juan Manuel Sosa, "Activismo judicial de los tribunales constitucionales y su posible legitimación a través de mecanismos vinculados a la democracia deliberativa”, acceso el 10 de enero de 2019,

http://www.conpedi.org.br/wp-content/uploads/2017/08/Juan-Manuel-Sosa-Sacio-Peru.pdf

21 Pedro Grández, El Tribunal Constitucional y argumentación jurídica (Lima: Palestra Editores, 2010), 70-71.

22 Juan Manuel Sosa, partiendo de la noción de "constitucionalismo fuerte" planteada por Jeremy Waldron, plantea que en la práctica los jueces activistas tienden a: "(1) dictar decisiones obligatorias-directivas; (2) dictar decisiones en cierta medida invasivas de competencias ajenas (o incluso a atribuirse nuevas competencias); (3) ser creativos en sus respuestas e innovar en el ordenamiento jurídico; (4) dictar decisiones con alcances generales e incluso estructurales; y (5) tener la última palabra en materia de interpretación constitucional". Juan Manuel Sosa. "Activismo judicial de los tribunales constitucionales y su posible legitimación a través de mecanismos vinculados a la democracia deliberativa", acceso el 10 de enero de 2019, http://www.conpedi.org.br/wp-content/uploads/2017/08/Juan-Manuel-Sosa-Sacio-Peru.pdf 
Cualquier práctica de interpretación y aplicación de las reglas jurídicas a la que se imprima una orientación correctora supone por principio una extralimitación de la función judicial (esto es, aquello que no podría hacer legítimamente un juez) si ello supone ir más allá del sentido textual o a lo sumo de la intención objetiva del legislador encerrada en esas reglas. $\mathrm{El}$ activismo es, pues —entendido en sentido heterointegrador y no autointegrador- un fenómeno fundamentalmente patológico: queda proscrito como una desviación perniciosa de la función judicial, su desnaturalización en realidad. ${ }^{23}$

Sin embargo, esta concepción al no corresponder con los caracteres del proceso de constitucionalización del derecho, como es la presencia de principios que exigen a los jueces la interpretación de las reglas a la luz de aquellas directrices que buscan optimizar la validez material de los derechos, ${ }^{24}$ resulta bastante débil en el contexto actual, toda vez que la mayoría de Estados donde han surgido prácticas activistas se afirman (o buscan afirmarse) como Estados Constitucionales de Derecho.

Por otro lado, desde la teoría de la democracia, la legitimidad de estas prácticas es cuestionada al punto de plantear una relación dicotómica entre activismo judicial y gobierno democrático. ${ }^{25}$ Algunas de las objeciones planteadas a la legitimidad de las prácticas relativas al control de la constitucionalidad — que se han agudizado frente a las nuevas formas de activismo- se pueden resumir en las siguientes preguntas:

¿Por qué la democracia constitucional, cuya mayor expresión empírica puede ser expresada en el voto libre y voluntario de los ciudadanos, debe, no obstante, merecer constantes controles cuando no verdaderas sanciones de parte de un reducido grupo de seńores de toga? ¿Han devenido los tribunales en un poder antidemocrático que, antes que fortalecer las instituciones de la democracia, atacan por el contrario el centro mismo de la democracia con su juicio sobre la Ley? ${ }^{26}$

En efecto, el carácter contramayoritario del Poder Judicial lo coloca en una posición en la que si su actuar es percibido como el ejercicio de una función que le corresponde a cualquier de los otros poderes, se ve en serios problemas, toda vez que aquellas poseen mayor grado de representatividad (el Parlamento como el caso más evidente). Por otro lado, si no son cuestionadas desde el punto de vista de la representatividad, son cuestionadas desde el punto de vista de la participación de la sociedad civil en las cuestiones públicas (como aquellas que se discuten en las altas cortes relativas a las políticas públicas).

\footnotetext{
23 Jesús Vega, "Límites de la jurisdicción, concepciones del derecho y activismo judicial”, Doxa 41 (2018): 125.

24 Jesús Vega, "Límites de la jurisdicción, concepciones del derecho y activismo judicial”, Doxa 41 (2018): 135.

25 Hans-Rudolf Horn, "Activismo judicial versus gobierno democrático", Anuario Iberoamericano de Justicia Constitucional 14 (2010).

26 Pedro Grández, El Tribunal Constitucional y argumentación jurídica (Lima: Palestra Editores, 2010), 66-67.
} 
Esta situación obliga a elaborar estrategias para enfrentar estos cuestionamientos, pues si bien los efectos positivos que puedan tener sus decisiones en grandes sectores de la población pueden generar respaldo popular, esta situación no anula por sí misma las razones provenientes desde la crítica, toda vez que se basan en la misma lógica del sistema establecido de frenos y contrapesos. Es así que de entre las estrategias de legitimación de dichas decisiones que han surgido, existen propuestas que pretenden conciliar por un lado formas de constitucionalismo fuerte, ${ }^{27}$ y por el otro la participación popular, inspirada en las teorías deliberativas de la democracia la cual plantea como criterio de legitimidad de las decisiones la participación en condiciones de igualdad de todos los potencialmente afectados por ella. ${ }^{28}$ De esta manera, surge el denominado "constitucionalismo dialógico", caracterizado por incluir arreglos institucionales como las audiencias públicas, amicus curiae, acciones de legitimación colectiva, elección popular de los jueces, la consulta previa, el control judicial respecto al proceso de creación legislativa, litigios de reforma estructural, sentencias exhortativas, monitoreos y mecanismos de seguimiento periódico respecto a la implementación de las decisiones judiciales, entre otros mecanismos. ${ }^{29}$

\section{CONCLUSIONES}

Las cortes constitucionales en América Latina, a través de mecanismos como la técnica de Estado de Cosas Inconstitucionales aplicada en las denominadas sentencias estructurales, han permitido develar las deficiencias institucionales de las entidades públicas respecto a la garantía de los derechos fundamentales. De esta manera, la elaboración de los Planes Nacionales de Derechos Humanos han reforzado el rol del juez constitucional ya no solo como promotor de políticas públicas a través de sus declaraciones, sino lo ha convertido en un actor importante en las diversas etapas interviniendo con el fin de verificar el cumplimiento de los estándares constitucionales establecidos en la sentencia en cada etapa del proceso de formulación de dichas políticas.

Sin embargo, la intervención de las cortes constitucionales en la formulación de políticas públicas con enfoque basado en derechos humanos, al ir más allá de la identificación de una situación inconstitucional que vulnera los derechos fundamentales, puede cuestionarse desde diversas ópticas respecto a su legitimidad democrática. Es por ello que resulta importante el desarrollo de "estrategias de legitimación" como las propuestas por el denominado constitucionalismo dialógico, las cuales buscan potenciar el diálogo entre las entidades estatales y la sociedad civil, participando los jueces como articuladores del diálogo público.

27 Waldron se refiere a estos modelos como aquellos donde las Cortes tienen el poder de hacer inoperable la norma que ha sido declarada como inconstitucional. Jeremy Waldron, "Control de constitucionalidad y legitimidad política", Dikaion 27, $\mathrm{N}^{\circ} 1$ (2018): 12.

28 Luciano Nosetto, "Legitimidad democrática y judicialización de la política: el caso de la Argentina contemporánea", Ivs Fvgit 21 (2018): 153.

29 Carlos Giuffré, "Democracia deliberativa y surgimiento del constitucionalismo dialógico”, Ética y Discurso, 3, $N^{\circ}$ 1(2018): 47. 


\section{REFERENCIAS}

- Banco Interamericano de Desarrollo. La politica de las políticas públicas. México D.F.: Editorial Planeta, 2006.

- Bregaglio, Renata; Constantino, Renato y Carmela Chávez. Políticas públicas con enfoque de derechos humanos en el Perú. Lima: IDEHPUCP - Konrad Adenauer, 2014.

- Carilloclla, Pavel. La justicia constitucional y las politicas públicas: una aproximación. Santiago de Chile: Universidad Diego Portales, 2008.

- Giuffré, Carlos. "Democracia deliberativa y surgimiento del constitucionalismo dialógico". Ética y Discurso 3, No 1 (2018): 35-60.

- Grández, Pedro. El Tribunal Constitucional y argumentación jurídica. Lima: Palestra Editores, 2010.

- Horn, Hans-Rudolf. "Activismo judicial versus gobierno democrático". Anuario Iberoamericano de Justicia Constitucional 14 (2010): 183-192.

- Landa Arroyo, César. Organización y funcionamiento del Tribunal Constitucional: entre el derecho y la política. Lima: Palestra, 2011.

- Landa Arroyo, César. "Justicia constitucional en América Latina”. Revista Ius et veritas 49 (2014):108-117.

- Ministerio de Justicia y Derechos Humanos del Perú. Plan Nacional de Derechos Humanos 2018-2021. Acceso el 10 de enero de 2019. http://spij.minjus.gob.pe/content/banner_secundario/img/muestra/PLAN-ANUAL.pdf

- Nosetto, Luciano. "Legitimidad democrática y judicialización de la política: el caso de la Argentina contemporánea”. Ivs Fvgit 21 (2018): 145-166.

- Oficina del Alto Comisionado para los Derechos Humanos. Preguntas frecuentes sobre el enfoque de derecho en la cooperación para el desarrollo. Nueva York y Ginebra: OACHD, 2006.

- Quinche-Ramírez, Manuel y Juan Rivera-Rugeles. "El control judicial de las políticas públicas como instrumento de inclusión de los Derechos Humanos". Vniversitas 121 (2010): 113-138.

- Quiroga, Aníbal. "La Justicia Constitucional”, Revista Derecho PUCP 41 (1987): 323351.

- Rivera, José. "Fundamentos sobre el carácter vinculante de las resoluciones del Tribunal Constitucional". Anuario Iberoamericano de Justicia Constitucional 9 (2005): 343-356. 
- Santiago, Alfonso. "Neoconstitucionalismo". En Anales de la Academia de Ciencias Morales y Políticas - Instituto de Política Constitucional. Buenos Aires: Instituto de Política Constitucional, 2008: 3-26.

- Sosa, Juan Manuel. "Activismo judicial de los tribunales constitucionales y su posible legitimación a través de mecanismos vinculados a la democracia deliberativa”. Acceso el 10 de enero de 2019.

http://www.conpedi.org.br/wp-content/uploads/2017/08/Juan-Manuel-Sosa-Sacio-Peru.pdf

- Steiner, Christian. "Presentación”, en Justicia Constitucional y Derechos Fundamentales $N^{\circ}$ 5: La protección de los derechos sociales, las sentencias estructurales, ed. por Víctor Bazán. Bogotá: Konrad Adenauer, 2015.

- Sumaria, Omar y Karla Vasallo. "Un nuevo actor político: la participación de los tribunales constitucionales en la elaboración de las políticas públicas". Revista Derecho y Sociedad 40 (2013): 121-131.

- Torres-Melo, Jaime y Jairo Santander. Introducción a las politicas públicas: conceptos y herramientas desde la relación entre Estado y ciudadanía. Bogotá: IEMP Ediciones, 2013.

- Vega, Jesús. "Límites de la jurisdicción, concepciones del derecho y activismo judicial”. Doxa 41 (2018): 123-150.

- Waldron, Jeremy. "Control de constitucionalidad y legitimidad política". Dikaion 27, $\mathrm{N}^{\circ} 1$ (2018): 7-28.

http://doi.org/10.5294/dika.2018.27.1.1

RECIBIDO: 08/07/2019

APROBADO: $15 / 11 / 19$ 